\title{
Early maladaptive schemas and suicidal ideation in depressed patients
}

\author{
Flink, N.
}

2017

Flink, N , Lehto , S M , Koivumaa-Honkanen, H , Viinamäki , H, Ruusunen , A ,

Valkonen-Korhonen, M \& Honkalampi , K 2017 , ' Early maladaptive schemas and suicidal

ideation in depressed patients ' , European Journal of Psychiatry , vol. 31 , no. 3 , pp. 87-92 . https://doi.org/10.1016

http://hdl.handle.net/10138/299537

https://doi.org/10.1016/j.ejpsy.2017.07.001

publishedVersion

Downloaded from Helda, University of Helsinki institutional repository.

This is an electronic reprint of the original article.

This reprint may differ from the original in pagination and typographic detail.

Please cite the original version. 


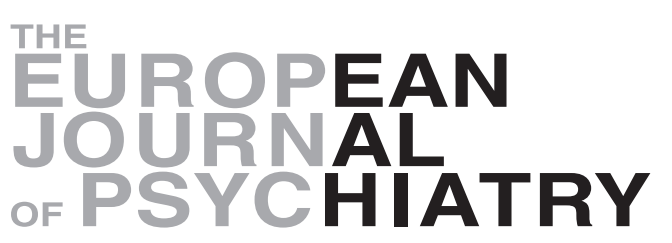

www.elsevier.es/ejpsy

\title{
ORIGINAL ARTICLE
}

\section{Early maladaptive schemas and suicidal ideation in depressed patients}

\author{
N. Flink ${ }^{a, *}$, S.M. Lehto ${ }^{b, c, d}$, H. Koivumaa-Honkanen ${ }^{b, c, e, f, g, h}$, H. Viinamäki ${ }^{b, c}$, \\ A. Ruusunen ${ }^{c}, M$. Valkonen-Korhonen ${ }^{b, c}$, K. Honkalampi ${ }^{a}$ \\ a School of Educational Sciences and Psychology, University of Eastern Finland, P.O. Box 111, 80101 Joensuu, Finland \\ b Institute of Clinical Medicine, University of Eastern Finland, P.O. Box 1627, FI-70211 Kuopio, Finland \\ c Department of Psychiatry, Kuopio University Hospital, P.O. Box 100, FI-70029 KYS Kuopio, Finland \\ d Department of Psychology and Logopedics, Faculty of Medicine, University of Helsinki, P.O. Box 63, 00014 University of Helsinki, \\ Finland \\ e South-Savonia Hospital District, Mikkeli, Finland \\ ${ }^{f}$ North Karelia Central Hospital, Joensuu, Finland \\ g SOTE, lisalmi, Finland \\ ${ }^{\mathrm{h}}$ Lapland Hospital District, Rovaniemi, Finland
}

Received 16 January 2017; accepted 18 July 2017

Available online 12 August 2017

\section{KEYWORDS}

Early maladaptive schemas;

Depression;

Suicidal ideation

\begin{abstract}
Background and objectives: Suicidal ideation is a key risk factor for suicidal behaviour among depressed individuals. To explore underlying cognitive patterns associated with suicidal ideation, the present study compared early maladaptive schemas (EMSs) among psychiatric outpatients in treatment for major depressive disorder with and without current suicidal ideation. Methods: The sample consisted of 79 depressed patients who responded to the background questionnaire and completed the Young Schema Questionnaire short form-extended, 21-item Beck Depression Inventory and Beck Hopelessness Scale.

Results: Patients with suicidal ideation were more maladaptive in respect to the majority of EMSs compared to those without. After controlling for the concurrent depressive symptom severity and hopelessness 'Vulnerability to Harm or Illness' EMS, which concerns catastrophising beliefs, remained a predictor for suicidal ideation.

Conclusion: EMSs may contribute to suicidal ideation among depressed individuals regardless of their mood and future orientation. These results offer implications for the assessment and treatment of suicidality.

(c) 2017 Asociación Universitaria de Zaragoza para el Progreso de la Psiquiatría y la Salud Mental. Published by Elsevier España, S.L.U. All rights reserved.
\end{abstract}

* Corresponding author.

E-mail address: nflink@uef.fi (N. Flink). 


\section{Introduction}

The association between depression, suicidal behaviours and suicidal ideation has been documented extensively. ${ }^{1-3}$ Over half the patients with major depressive disorder (MDD) have suicidal ideation and suicidal thoughts tend to be persistent. ${ }^{3,4}$ Psychological factors related to suicidal ideation among depressed individuals have received less attention from researchers than nonfatal suicidal attempts or behaviours. Better knowledge of underlying factors in suicidal ideation could aid in suicide prevention, since suicidal ideation often precedes suicidal behaviour among depressed individuals. ${ }^{3,5}$ In particular, the severity of depressive symptoms and hopelessness has been linked with suicidal ideation. ${ }^{2,3,6}$ However, several other factors, such a history of nonadherence, low levels of social and occupational functioning, low social support and reluctance to admit mental health problems also contribute to suicidal ideation. $3,6,7$

Exploring underlying cognitive patterns could offer further insight into the relationship between suicidal ideation and depression. Recent studies have associated early maladaptive schemas (EMSs) with both depression ${ }^{8-10}$ and suicidal behaviours in clinical populations. ${ }^{11-13}$ In schema theory ${ }^{14}$ EMSs are defined as stable, trait-like selfperpetuating cognitive patterns that influence thinking and behaviour in a dysfunctional way. EMSs are theorised to emerge from unmet basic needs and traumatic experiences during childhood, combined with the individual's emotional temperament; when triggered, schemas dominate thoughts and feelings, leading to negative emotions and dysfunctional thoughts. ${ }^{14}$ A total of 18 EMSs have been defined, which are further categorised under five broader schema domains and can be measured using the Young Schema Questionnaire. ${ }^{14}$ Brief descriptions of EMS domains are presented in Table 1.

Schemas are thought to represent the deepest level of dysfunctional cognitions. ${ }^{14}$ In accordance with the schema theory, EMSs have demonstrated high stability over time in depressed patients also after controlling for severity of depression. ${ }^{8,9}$ The EMSs from the 'Disconnection and Rejection' and 'Impaired Autonomy and Performance' schema domains in particular have been associated with depression. ${ }^{8-10}$ Depression-related EMSs are consistent with the cognitive model of depression, and place beliefs of failure, loss, and worthlessness at the core of depressive symptoms. ${ }^{8}$ Even though studies on clinical populations indicate associations between EMSs and suicidality, ${ }^{11,12}$ research focusing on suicidal ideation and EMSs has been sparse. Previously EMSs have been shown to correlate with suicidal ideation in a sample of chronically traumatised patients. ${ }^{13}$ To the best of our knowledge no study has explored the associations between EMSs and suicidal ideation in depressed patients, or in respect to concurrent depressive symptom severity and hopelessness. Given that both EMSs and suicidal ideation are associated with the severity of depressive symptoms, ${ }^{2,6,8,10}$ we hypothesised that suicidal ideation would be closely connected to depression-related EMSs.

The aim of the present exploratory study was to assess 1) if EMSs among psychiatric outpatients in treatment for MDD differ by presence of current suicidal ideation, 2) if EMSs associated with suicidal ideation are similar to those previously associated with depression, and 3) whether specific
Table 1 Definitions of early maladaptive schema (EMS) domains by Young and colleagues. ${ }^{14}$

1) Disconnection and Rejection domain: Schemas involving expectations that one's needs for security, safety, and stability will not be met. Includes EMSs:

Abandonment/Instability, Mistrust/Abuse, Emotional Deprivation, Defectiveness/Shame and Social Isolation/Alienation

2) Impaired Autonomy and Performance: Schemas involving expectations that one's ability and capacity to separate, survive, cope independently, or perform successfully will be impaired. Includes EMSs: Dependence/Incompetence, Vulnerability to Harm or Illness, Enmeshment/Underdeveloped Self and Failure

3) Impaired Limits: Schemas involving difficulties in setting internal limits, feeling responsibility, or setting long-term goals. Includes EMSs: Entitlement/Grandiosity and Insufficient Self-control/Self-discipline

4) Other-directedness: Schemas where others' needs, desires, or responses are respected and taken into account at the expense of one's own needs. Includes EMSs: Subjugation, Self-sacrifice and Approval-seeking/Recognition-seeking

5) Overvigilance and Inhibition: Schemas where spontaneous feelings and impulses are suppressed and replaced by rigid, internalised rules about performance and behaviour. Includes EMSs: Negativity/Pessimism, Emotional Inhibition, Unrelenting Standards/Hypercriticalness and Punitiveness

EMSs predict suicidal ideation when the effects of severity of depression and hopelessness are taken into account.

\section{Methods}

\section{Participants and procedures}

The present study was based on data from a naturalistic follow-up study on psychiatric outpatients with MDD (aged 18-65 years) recruited from the Department of Psychiatry at Kuopio University Hospital. At baseline, the diagnosis of MDD was confirmed by trained mental health professionals using the Structured Clinical Interview for DSM-IV Axis I Disorders (SCID-I). ${ }^{15}$ Patients suffering from epilepsy, bipolar disorder, psychotic depression or other psychotic disorders and depression related directly to somatic conditions or substance abuse were excluded from the study. Of the initial 100 patients, 79 (58.2\% women) participated to the present study phase, i.e., 5-13 months after the baseline assessment (mean 8.40 months, SD 2.30). We observed no differences in gender $(p=0.630)$, marital status $(p=0.594)$, severity of depression $(p=0.585)$ or age at first depressive episode $(p=0.663)$ between participants of the present study phase and non-participants. Participants were older than non-participants (mean age 40.53 years, SD 11.73 vs. 34.29 years, SD $12.20, p=0.034$ ). At the time of the present study phase, SCID-I was repeated and participants completed the study questionnaire and measurements for EMSs, depression and hopelessness. Out of 
the 79 patients, $57.7 \%$ met diagnostic criteria for MDD, and 42.3\% were in full or partial remission according to SCID-I. All participants provided written informed consent before entering the study. The study protocol was accepted by the Research Ethics Committee of the Northern Savo Hospital District.

\section{Measures}

The study questionnaire gathered information on demographic variables and illness history (sex, age, marital status, age at first depressive episode and number of lifetime MDD episodes). The Young Schema Questionnaire short form-extended (YSQ-S2-extended) was used to measure 18 different EMSs. The questionnaire contains 90 selfstatements (five for each schema), which respondents are asked to rate on a Likert scale ranging from 1 (completely untrue) to 6 (describes perfectly). EMS subscales were used as continuous variables based on the mean of the five schema statements. The factor structure and reliability of the Finnish version of the YSQ-S2-extended have been established by Saariaho et al. ${ }^{16}$ In the present study, the internal reliability coefficient alpha ranged from 0.74 to 0.95 for all 18 EMS subscales (median $\alpha=.88$ ).

The 21-item version of the Beck Depression Inventory $(B D I-21)^{17}$ was used to measure the severity of depression. One of its items (i.e., number 9) concerning suicidal thoughts and wishes was used to assess suicidal ideation. Absence of suicidal ideation was based on the response alternative, 'I don't have any thoughts of killing myself', and current suicidal ideation was based on the following three responses: 1) 'I have thoughts of killing myself, but I would not carry them out'; 2) 'I would like to kill myself'; and 3) 'I would kill myself if I had the chance'. Thus, suicidal ideation was defined in agreement with Diekstra and Garnefski ${ }^{18}$ as cognitions varying from transient thoughts about the worthlessness of life and death wishes to concrete plans for killing oneself and obsessive preoccupation with selfdestruction. In calculating BDI scores, the suicidality item was excluded (BDI range 0-60). Hopelessness was measured using the Beck Hopelessness Scale ${ }^{19}$ (HS), which is a 20-item rating scale measuring negative expectancies concerning oneself and one's future life. The total score of HS ranges from 0 to 20, with higher score indicating higher level of hopelessness.

\section{Data analysis}

Differences between patients with and without suicidal ideation were examined using the $\chi^{2}$ test for categorical variables and the $t$ test for continuous variables. The magnitude of difference in the EMS subscale scores was calculated using Cohen's $d$ as a measure of effect sizes (small if $d=0.2$, medium if $d=0.5$ and large if $d=0.8$ ). ${ }^{20}$ Patients with suicidal ideation were used as a reference group with positive or negative effect sizes indicating, respectively, higher or lower EMS scores in this group. Each EMS subscale found to be significantly associated with suicidal ideation in univariate analysis was further analysed in multivariate logistic models. In additional analyses, any of the demographic and clinical variables that differed significantly between the two groups were used as covariates due to their connection with suicidal ideation. All statistical tests were two-tailed, and analyses were conducted with the SPSS statistical package $\left(\right.$ IBM $^{\circledR}$ SPSS $^{\circledR}$ Statistics version 22.0).

\section{Results}

A total of $60.8 \%$ of patients suffered from current suicidal ideation. The demographic characteristics and clinical variables between patients with and without suicidal ideation are presented in Table 2 . There were no statistically significant differences between the groups in terms of age, gender, marital status, the mean age at the first depressive episode or the number of lifetime depressive episodes. Those with current suicidal ideation were significantly more depressed (BDI means 27.7 (SD 9.7) vs. $14.3(10.8), t=5.50, p=<0.001$ ) and hopeless (HS means 12.6 (SD 5.6) vs. 6.3 (4.4) $t=5.34$, $p=<0.001)$ than those without.

Patients with suicidal ideation scored significantly higher on 11 out of the 18 EMS subscales with at least moderate effect sizes (Table 3). The largest differences in EMS subscales between the groups were in 'Vulnerability to Harm or Illness' and 'Dependence/Incompetence' EMSs from 'Impaired Autonomy and Performance' schema domain and 'Defectiveness/Shame' and 'Social isolation/Alienation' EMSs from 'Disconnection/Rejection' schema domain. Moderate differences were observed also in EMSs from 'Other-Directedness' and 'Overvigilance and Inhibition' schema domains.

Because those with suicidal ideation were significantly more depressed and hopeless, BDI and HS scores were included as covariates in logistic models with significant EMS subscales as predictors of suicidal ideation. In the logistic models with BDI and HS, only the EMS subscale 'Vulnerability to Harm or Illness' remained statistically significant in respect to suicidal ideation $(p=0.037)$. This particular model was statistically significant $\left(\chi^{2}(3)=32.31, p=0.049\right)$ explaining $48.6 \%$ (Nagelkerke $R^{2}$ ) of the variance in suicidal ideation and correctly classified $88.9 \%$ of cases. Neither $\mathrm{BDI}(p=0.127)$ or $\mathrm{HS}(p=0.378)$ were significant in this model.

\section{Discussion}

A wide range of subscales for early maladaptive schemas were associated with suicidal ideation among depressed patients, but only 'Vulnerability to Harm or Illness' EMS predicted current suicidal ideation independently from depression and hopelessness. Given the exploratory nature of the present study, these findings should be considered as preliminary and need to be replicated in larger samples. However, results support prior research suggesting that EMSs may relate to suicidality, ${ }^{11-13}$ while the role of depressive symptoms and hopelessness in suicidal ideation has already been established. ${ }^{3-5}$

The 'Vulnerability to Harm or Illness' subscale concerns catastrophising beliefs and exaggerated fears of irrationally negative forecasted life-events (such as imminent medical, emotional or external catastrophes). ${ }^{14}$ Our results suggest, that catastrophising core beliefs could act as an 
Table 2 Demographic and clinical characteristics of depressed patients by suicidal ideation.

\begin{tabular}{|c|c|c|c|}
\hline & With suicidal ideation $(n=48)$ & Without suicidal ideation $(n=31)$ & Statistics \\
\hline \multicolumn{4}{|l|}{ Demographics } \\
\hline Gender (\% women) & $64.6 \%$ & $48.4 \%$ & $\chi^{2}=2.03, p=0.154$ \\
\hline Mean age $(S D)$ in years & $42.4(11.0)$ & $39.4(12.8)$ & $t=1.12, p=0.267$ \\
\hline Marital status (\% of total) & & & $\chi^{2}=1.95, p=0.377$ \\
\hline Single & $37.5 \%$ & $32.3 \%$ & \\
\hline Married & $43.8 \%$ & $58.1 \%$ & \\
\hline Divorced & $18.8 \%$ & $9.7 \%$ & \\
\hline \multicolumn{4}{|l|}{ Clinical characteristics } \\
\hline Mean BDI-21 (SD) & $27.7(9.7)$ & $14.3(10.8)$ & $t=5.50, p=<0.001$ \\
\hline Mean HS (SD) & $12.6(5.6)$ & $6.3(4.4)$ & $t=5.34, p=<0.001$ \\
\hline Mean age (SD) at first depr. episode & $27.1(13.5)$ & $27.6(14.4)$ & $t=-0.17, p=0.867$ \\
\hline Lifetime MDD episodes (\% of total) & & & $\chi^{2}=0.21, p=0.977$ \\
\hline One episode & $39.6 \%$ & $35.5 \%$ & \\
\hline Two episodes & $16.7 \%$ & $16.1 \%$ & \\
\hline Three episodes & $18.8 \%$ & $19.4 \%$ & \\
\hline Four or more episodes & $25.0 \%$ & $29.0 \%$ & \\
\hline
\end{tabular}

Note: SD, standard deviation; BDI-2I, 21 -item Beck Depression Inventory (item 9 omitted, range 0-60); HS, Beck Hopelessness Scale.

Table 3 Early Maladaptive Schema subscales ${ }^{\mathrm{a}}$ in depressed patients by suicidal ideation.

\begin{tabular}{|c|c|c|c|c|}
\hline Early Maladaptive Schema & $\begin{array}{l}\text { With suicidal } \\
\text { ideation }(n=48) \\
\text { Mean }(S D)\end{array}$ & $\begin{array}{l}\text { Without suicidal } \\
\text { ideation }(n=31) \\
\text { Mean (SD) }\end{array}$ & $p$ & $d$ \\
\hline \multicolumn{5}{|l|}{ Disconnection and Rejection domain } \\
\hline Abandonment/Instability & $3.2(1.5)$ & $2.6(1.5)$ & 0.071 & 0.40 \\
\hline Mistrust/Abuse & $3.0(1.3)$ & $2.1(1.1)$ & 0.002 & 0.75 \\
\hline Emotional Deprivation & $3.8(1.3)$ & $2.8(1.5)$ & 0.004 & 0.71 \\
\hline Defectiveness/Shame & $3.1(1.5)$ & $2.0(1.0)$ & $<0.001$ & 0.86 \\
\hline Social Isolation/Alienation & $3.7(1.4)$ & $2.4(1.4)$ & $<0.001$ & 0.93 \\
\hline \multicolumn{5}{|l|}{ Impaired Autonomy and Performance domain } \\
\hline Dependence/Incompetence & $2.8(1.1)$ & $1.7(0.9)$ & $<0.001$ & 1.09 \\
\hline Vulnerability to Harm or Illness & $3.1(1.2)$ & $1.7(1.0)$ & $<0.001$ & 1.27 \\
\hline Enmeshment/Underdeveloped Self & $2.0(1.1)$ & $1.5(0.8)$ & 0.062 & 0.52 \\
\hline Failure & $3.3(1.6)$ & $2.5(1.5)$ & 0.026 & 0.52 \\
\hline \multicolumn{5}{|l|}{ Impaired Limits domain } \\
\hline Entitlement/Grandiosity & $1.9(0.8)$ & $1.7(0.7)$ & 0.127 & 0.27 \\
\hline Insufficient Self-control/Self-discipline & $2.7(0.9)$ & $2.3(1.1)$ & 0.079 & 0.40 \\
\hline \multicolumn{5}{|l|}{ Other-Directedness domain } \\
\hline Subjugation & $2.5(1.1)$ & $1.9(1.0)$ & 0.011 & 0.57 \\
\hline Self-sacrifice & $4.0(1.2)$ & $3.4(1.1)$ & 0.047 & 0.52 \\
\hline Approval-seeking/Recognition-seeking & $3.5(1.0)$ & $3.2(1.2)$ & 0.331 & 0.27 \\
\hline \multicolumn{5}{|l|}{ Overvigilance and Inhibition domain } \\
\hline Negativity/Pessimism & $4.1(1.1)$ & $3.1(1.4)$ & 0.001 & 0.79 \\
\hline Emotional Inhibition & $2.6(1.4)$ & $2.3(1.2)$ & 0.296 & 0.23 \\
\hline Unrelenting Standards/Hypercriticalness & $3.8(1.3)$ & $3.3(1.3)$ & 0.073 & 0.38 \\
\hline Punitiveness & $3.6(1.3)$ & $2.6(1.3)$ & 0.001 & 0.77 \\
\hline
\end{tabular}

a Subscales of the Young Schema Questionnaire short form-extended (YSQ-S2-extended).

independent risk factor for suicidal ideation among depressed individuals. Suicidality and suicidal ideation have been proposed to represent a particular pattern of information processing, which arises from feelings of entrapment and a sense of no escape. ${ }^{21}$ Theoretically, fear of unpreventable catastrophes could create a sense of no control over one's life, which could induce hopelessness, depressed mood and suicidal thought processes.

In general, patients with suicidal ideation had consistently higher subscale scores in the majority of EMSs in 
the domains of 'Disconnection and Rejection' and 'Impaired Autonomy and Performance'. These domains deal with beliefs related to inadequate acceptance or security and impaired ability to survive and cope. ${ }^{14}$ Moderate differences were observed in the EMS subscales of 'Subjugation' and 'Self-sacrifice' from the 'Other-Directedness' domain and in 'Negativity/Pessimism' and 'Punitiveness' from the 'Overvigilance and Inhibition' domain. The first domain places excessive focus on feelings of others, and the second overemphasises suppression of one's feelings. ${ }^{14}$ Higher EMS subscale scores observed among those with suicidal ideation were similar to those previously associated with severity of depressive symptoms ${ }^{8-10,22}$ and support our hypothesis that suicidal ideation in depressed patients is closely connected to more general depression-related maladaptive cognitive patterns.

Schema theory proposes that EMSs rise from traumatic experiences and unmet needs during childhood and adolescence. ${ }^{14}$ Considering the notable stability of depression-related EMSs $^{9}$ and strong connection between childhood adversities, depression ${ }^{23}$ and suicidality, ${ }^{24}$ high EMS scores in patients with suicidal ideation may be related to earlier traumatic life experiences. Further research should focus on clarifying possible mechanisms between early life adversities, EMSs, suicidal ideation and depression.

There are important limitations to this study that should be considered. First, suicidal ideation was assessed using single question instead of a scale or structured interview. Second, cross-sectional study setting does not allow causal conclusions. Third, sample size was relatively small, which may affect the generalisability of our findings. Therefore, further replications with larger samples and structured assessment of suicidal ideation are needed.

In conclusion, the present study showed that catastrophising cognitive schema may be of particular importance in suicidal ideation among depressed patients. Furthermore, suicidal ideation was found to be associated with several maladaptive cognitive patterns, which are connected to concurrent depressive symptom severity and hopelessness. From a clinical perspective, assessing EMSs may be especially relevant when psychological factors related to suicidal ideation are been identified. Results also highlight the possibility that, in addition to targeting depression and hopelessness, focusing interventions on catastrophising cognitive patterns could be beneficial when treating suicidality among depressed patients. In order to apply these findings into clinical practice, further replications and expanding of the results are, however, needed.

\section{Funding}

SML was supported by a grant from the Paulo Foundation.

\section{Conflicts of interest}

Authors none were declared.

\section{References}

1. Arsenault-Lapierre G, Kim C, Turecki G. Psychiatric diagnoses in 3275 suicides: a meta-analysis. BMC Psychiatry. 2004;4:37.

2. Hintikka J, Viinamäki H, Koivumaa-Tanskanen H-T, Saarinen P, Tanskanen A, Lehtonen J. Risk factors for suicidal ideation in psychiatric patients. Soc Psychiatry Psychiatr Epidemiol. 1998; 33:235-40.

3. Sokero TP, Melartin TK, Rytsälä HJ, Leskelä US, Lestelä-Mielonen PS, Isometsä ET. Suicidal ideation and attempts among psychiatric patients with major depressive disorder. J Clin Psychiatry. 2003;64:1094-100.

4. Hintikka J, Pesonen T, Saarinen P, Tanskanen A, Lehtonen J, Viinamäki H. Suicidal ideation in the Finnish general population. A 12-month follow-up study. Soc Psychiatry Psychiatr Epidemiol. 2001;36:590-4.

5. Kessler RC, Borges G, Walters EE. Prevalence of and risk factors for lifetime suicide attempts in the National Comorbidity Survey. Arc Gen Psychiatry. 1999;56:617-26.

6. Ando S, Kasai K, Matamura M, Hasegawa Y, Hirakawa H, Asukai N. Psychosocial factors associated with suicidal ideation in clinical patients with depression. J Aff Dis. 2013;151:561-5.

7. Pompili M, Venturini P, Palermo M, Stefani $H$, Seretti ME, Lamis DA, et al. Mood disorders medications: predictors of nonadherence - review of the current literature. Expert Rev Neurother. 2013;13:809-25.

8. Renner F, Lobbestael J, Peeters F, Arntz A, Huibers M. Early maladaptive schemas in depressed patients: stability and relation with depressive symptoms over the course of treatment. $J$ Affect Disord. 2012;136:581-90.

9. Wang CE, Halvorsen M, Eisemann M, Waterloo K. Stability of dysfunctional attitudes and early maladaptive schemas: a 9 year follow-up study of clinically depressed subjects. J Behav Ther Exp Psychiatry. 2010;41:389-96.

10. Halvorsen M, Wang CE, Richter J, Myrland I, Pedersen SK, Eisemann $M$, et al. Early maladaptive schemas, temperament and character traits in clinically depressed and previously depressed subjects. Clin Psychol Psychother. 2009;16:394-407.

11. Nilsson KK. Early maladaptive schemas in bipolar disorder patients with and without suicide attempts. J Nerv Ment Dis. 2016;204:236-9.

12. Pauwels E, Dierckx E, Schoevaerts K, Claes L. Early maladaptive schemas in eating disordered patients with or without nonsuicidal self-injury. Eur Eat Disorders Rev. 2016;24:399-405.

13. Dutra L, Callahan K, Forman E, Mendelsohn M, Herman J. Core schemas and suicidality in a chronically traumatized population. J Nerv Ment Dis. 2008;196:71-4.

14. Young JE, Klosko JS, Weishaar ME. Schema therapy: a practitioner's guide. New York, NY: Guilford Press; 2003.

15. First MB, Spitzer RL, Gibbon M, Williams JB. Structured clinical interview for DSM-IV axis I disorders - patient edition (SCID-I/P, version 2.0). New York: New York State Psychiatric Institute, Biometrics Research Department; 1996.

16. Saariaho TH, Saariaho AS, Karila IA, Joukamaa MI. The psychometric properties of the Finnish Young Schema Questionnaire in chronic pain patients and a non-clinical sample. J Behav Ther Exp Psychiatry. 2009;40:158-68.

17. Beck AT, Ward CH, Mendelson M, Mock J, Erbaugh J. An inventory for measuring depression. Arch Gen Psychiatry. 1961;4:561-71.

18. Diekstra RFW, Garnefski N. On the nature, magnitude, and causality of suicidal behaviors: an international perspective. Suicide Life Threat Behav. 1995;25:36-57.

19. Beck AT, Weissman A, Lester D, Trexler L. The measurement of pessimism: the Hopelessness Scale. J Consult Clin Psychol. 1974;42:861-5.

20. Cohen J. Statistical power analysis for the behavioral sciences. Hillsdale, NJ: Lawrence Erlbaum; 1988. 
21. Williams JMG. Suicide and attempted suicide. London, UK: Penguin; 2001.

22. Shah R, Waller G. Parental style and vulnerability to depression: the role of core beliefs. J Nerv Ment Dis. 2000;188:19-25.

23. Nanni V, Uher R, Danese A. Childhood maltreatment predicts unfavorable course of illness and treatment outcome in depression: a meta-analysis. Am J Psychiatry. 2012;2:141-51.
24. Dube SR, Anda RF, Felitti VJ, Chapman DP, Williamson DF, Giles WH. Childhood abuse, household dysfunction, and the risk of attempted suicide throughout the life span: findings from the Adverse Childhood Experiences Study. JAMA. 2001;286:3089-96. 